\title{
Dual $\mathrm{QED}_{3}$ at " $N_{F}=1 / 2$ " is an interacting CFT in the infrared
}

\author{
Dietrich Roscher, Emilio Torres and Philipp Strack \\ Institut für Theoretische Physik, Universität zu Köln, \\ D-50937 Cologne, Germany \\ E-mail: droscher@thp.uni-koeln.de, torres@thp.uni-koeln.de, \\ pstrack13@gmail.com
}

ABSTRACT: We study the fate of weakly coupled dual $\mathrm{QED}_{3}$ in the infrared, that is, a single two-component Dirac fermion coupled to an emergent U(1) gauge field, but without Chern-Simons term. This theory has recently been proposed as a dual description of $2 \mathrm{D}$ surfaces of certain topological insulators. Using the renormalization group, we find that the interplay of gauge fluctuations with generated interactions in the four-fermi sector stabilizes an interacting conformal field theory (CFT) with finite four-fermi coupling in the infrared. The emergence of this CFT is due to cancellations in the $\beta$-function of the four-fermi coupling special to " $N_{F}=1 / 2$ ". We also quantify how a possible "strong" Dirac fermion duality between a free Dirac cone and dual $\mathrm{QED}_{3}$ would constrain the universal constants of the topological current correlator of the latter.

Keywords: Duality in Gauge Field Theories, Spontaneous Symmetry Breaking, Topological States of Matter

ARXIV EPRINT: 1605.05347 


\section{Contents}

1 Introduction 1

1.1 Key results and outline of paper 2

2 Conformal scaling and its breakdown for $\mathrm{QED}_{3}$ with $N_{\mathrm{f}}^{(4)}$ 4-component fermions

2.1 Fierz-complete action 3

$2.2 \beta$-functions 4

2.3 Recap of interacting conformal fixed point for $N_{\mathrm{f}}^{(4)}>N_{\mathrm{f}, \mathrm{c}}^{(4)} \quad 5$

2.4 Breakdown criterion of conformal scaling at $N_{\mathrm{f}, \mathrm{c}}^{(4)} \quad 6$

3 Dual $\mathrm{QED}_{3}$ with a single 2-component fermion $N_{\mathrm{f}}^{(2)}=1 \quad 8$

3.1 Interacting conformal fixed point 8

$\begin{array}{ll}3.2 \text { Discussion } & 9\end{array}$

4 Implications of a possible "strong" Dirac fermion duality 9

$\begin{array}{lll}4.1 & \text { Constraining the topological current correlator } & 10\end{array}$

$\begin{array}{lll}4.2 & \text { Scaling dimension of composite mass operator at one-loop } & 11\end{array}$

5 Conclusions 13

$\begin{array}{lll}\text { A Derivation of } \beta \text {-function } \partial_{t} \lambda \text { for four-fermi coupling } \lambda & 14\end{array}$

B Cancellation of $\lambda^{2}$ term from flavor trace in $\partial_{t} \lambda \quad 15$

\section{Introduction}

There are several proposals claiming that a good starting point to describe more than one strongly interacting topological phase is dual $\mathrm{QED}_{3}$ : a single two-component (composite) Dirac fermion coupled to an emergent $\mathrm{U}(1)$ gauge field [1-3]:

$$
\mathcal{L}_{\mathrm{cf}}=\bar{\psi}(i \not \partial+\bar{e} \not \phi) \psi+\ldots
$$

Here $\bar{\psi}$ are not the fundamental electrons but two-component composite fermions. And $\bar{e}$ is not the physical, electric charge but the charge with respect to the emergent gauge field $a_{\mu}$. There is no Chern-Simons term for $a_{\mu}$ and this can be attributed to a restricted set of large gauge transformations. That is, only certain types of electric fluxes are allowed on the surface of the topological insulator [2]. A more complete and general derivation of various possibilities for gauge and symmetry transformations appeared shortly after completion of the present manuscript $[4,5]$. The dots stand for terms that are allowed by symmetries to 
be generated under a renormalization group $(R G)$ flow, such as four-fermi interactions and radiative corrections to the propagators.

In high-energy physics, Dirac fermions are typically collected in a 4D-representation of the fermion spinor algebra; the case of interest in the present paper therefore laxly corresponds to " $N_{F} \equiv N_{\mathrm{f}}^{(4)}=1 / 2$ ". It has not received much attention so far due to its novelty and only very recent association to topological condensed matter systems. Moreover, this case falls outside the region of validity of the Vafa-Witten theorem, which states that vector-like gauge theories cannot dynamically generate fermion masses in $\bar{\psi} \psi$ channels, thereby breaking time-reversal and space-reflection symmetry [6]. But their proof only holds for a number of two-component fermions $N_{\mathrm{f}}^{(2)} \geq 4$ and even.

More broadly, a Dirac fermion analog to the particle-vortex duality of bosons has been proposed [2] and an explicit construction of a possible "strong" duality of eq. (1.1) to a free Dirac cone of electrically charged electrons on the level of partition functions has been put forward in ref. [7].

It is fair to say that whether, and in what form, and on what energy scales this Dirac fermion duality holds is an open question with fundamental implications across a variety of physical systems. In particular, not much is known about the low-energy fate and ground state phase diagram of eq. (1.1), which we will refer to as dual $\mathrm{QED}_{3}$ in what follows.

In this paper, we want to start filling this knowledge gap and we ask if/under what conditions the low-energy dynamics of weakly coupled dual $\mathrm{QED}_{3}$, eq. (1.1), remains conformal and by which mechanism interactions can generate a mass for the (composite) fermions at low energies. To achieve this, we will adapt the symmetry-breaking analysis of $N_{\mathrm{f}}^{(4)}$ fourcomponent fermions coupled to a $\mathrm{U}(1)$ gauge field of ref. [8] to a single two-component $N_{\mathrm{f}}^{(2)}=1$ fermion and compute the low-energy fixed-point structure for an initially weakly coupled eq. (1.1). The question of (chiral) symmetry-breaking of $\mathrm{QED}_{3}$ has been tackled intensely and we refer to the Introduction and Bibliography of refs. [8-10] for an overview.

\subsection{Key results and outline of paper}

Our main result is that eq. (1.1), if initially weak-to-moderately coupled, flows toward an interacting conformal field theory (CFT) in the infrared in which generated four-fermi couplings attain finite values. In the condensed matter context of dual $\mathrm{QED}_{3}$ as a surface description for topological insulators, this implies that interactions preserve the gapless responses and the system remains a topological (semi-)metal also at lowest energies. This result is derived and presented in subsection 4.1 .

In light of strong (chiral and vector) symmetry-breaking tendencies for $\mathrm{QED}_{3}$ at singledigit flavor number $N_{\mathrm{f}}^{(4)}$ [8], this result may seem surprising. On the other hand, the $N_{\mathrm{f}}^{(2)}=1$ case does not have the full chiral symmetry to begin with, as it may be viewed to operate within one chiral sector. The number of symmetries that can be broken is now reduced and there remains essentially only one independent four-fermi coupling $(\lambda)$ of the associated Fierz algebra (see section 3). With this, we find that gauge fluctuations never destabilize the four-fermi sector toward symmetry-breaking sufficiently strongly; instead, the flow is always attracted toward an infrared stable fixed-point for the four-fermi coupling, which preserves the scaling/conformal symmetry of the gauge sector. Surprisingly, this is 
due to the absence of a $\lambda^{2}$ term in the $\beta$-function for the four-fermi coupling: the flavor trace carries $\mathrm{a} \sim\left(N_{\mathrm{f}}^{(2)}-1\right) \lambda^{2}$. This is what stabilizes the CFT at $N_{\mathrm{f}}^{(2)}=1$. Related cancellations of $\beta$-functions in the single-flavor case also appear in the Gross-Neveu model; in particular these also hold at higher loop orders [11-13].

We believe our results are not limited to the RG technique used. An $\epsilon$-expansion [14] around the weakly interacting Gaussian fixed-point in $D=4$ should recover the same physics.

In section 2, we recapitulate how related runaway flows of different physical origin have been detected in ref. [8]. We present the Fierz-complete action of $N_{\mathrm{f}}^{(4)} 4$-component Dirac fermions from which we project out the $\beta$-functions for a single two-component fermion, $N_{\mathrm{f}}^{(2)}=1$ in section 3 .

In section 4, we explore consequences of a possible strong Dirac fermion duality. We determine exactly the universal constant of the topological current correlator of eq. (1.1), an interacting theory, by relating it to the electromagnetic response of a free Dirac cone. Finally, in subsection 4.2 we point out the need to include the generated four-fermi coupling (and possibly other ingredients) in order to establish exponent identities for operator dimensions in compliance with the duality.

In section 5 , we conclude the paper. Details of a direct derivation of the $\beta$-function for the four-fermi coupling $\lambda$ are relegated to two appendices A, B.

\section{Conformal scaling and its breakdown for $\mathrm{QED}_{3}$ with $N_{\mathrm{f}}^{(4)} 4$-component fermions}

In ref. [8], $\beta$-functions for $\mathrm{QED}_{3}$ were calculated using a $4 \mathrm{D}$-reducible representation of the fermionic spinor fields. The main scope of this work was an investigation of the symmetry breaking patterns of $\mathrm{QED}_{3}$, including chiral channels, by detection of runaway flows for fermionic couplings caused by fixed point annihilation. To set the stage for dual $\mathrm{QED}_{3}$, we now recapitulate the key elements of this analysis.

\subsection{Fierz-complete action}

Based on the bare action of a Maxwell term for the photons coupled to a set of $N_{\mathrm{f}}^{(4)}$ flavors of 4-component Dirac fermions (flavor index implicit)

$$
S=\int \mathrm{d}^{3} x\left\{\bar{\psi}(i \not \partial+\bar{e} \not \phi) \psi+\frac{1}{4} F_{\mu \nu} F^{\mu \nu}\right\}
$$

the following Fierz-complete ansatz for the euclidean, scale $(k-)$ dependent effective action is sufficient to study symmetry-breaking into the complete set of all possible fermionic channels

$$
\begin{aligned}
\Gamma_{k}[\bar{\psi}, \psi, a]=\int \mathrm{d}^{3} x\left\{\overline { \psi } \left(i Z_{\psi} \not \partial\right.\right. & +\bar{e} \phi) \psi+\frac{Z_{a}}{4} F_{\mu \nu} F_{\mu \nu}+\frac{Z_{a}}{2 \xi}\left(\partial_{\mu} a_{\mu}\right)^{2} \\
& \left.+\frac{\tilde{\bar{g}}}{2 N_{\mathrm{f}}^{(4)}}\left(\bar{\psi} \gamma_{45} \psi\right)^{2}+\frac{\bar{g}}{2 N_{\mathrm{f}}^{(4)}}\left(\bar{\psi} \gamma_{\mu} \psi\right)^{2}\right\} .
\end{aligned}
$$


Here, for $\mu=1,2,3$,

$$
\begin{aligned}
& \gamma_{\mu}=i\left(\begin{array}{cc}
0 & -\sigma_{\mu} \\
\sigma_{\mu} & 0
\end{array}\right), \quad \gamma_{4}=\left(\begin{array}{ll}
0 & \mathbb{1} \\
\mathbb{1} & 0
\end{array}\right), \\
& \gamma_{5}=\gamma_{1} \gamma_{2} \gamma_{3} \gamma_{4}=\left(\begin{array}{cc}
\mathbb{1} & 0 \\
0 & -\mathbb{1}
\end{array}\right) \quad \text { and } \quad \gamma_{45}=i \gamma_{4} \gamma_{5}=i\left(\begin{array}{cc}
0 & -\mathbb{1} \\
\mathbb{1} & 0
\end{array}\right) \text {. }
\end{aligned}
$$

In the ansatz eq. (2.2), the last two terms $\tilde{\bar{g}}, \bar{g}$ are two four-fermi couplings from which all possible interaction channels, which can lead to condensation of fermion bilinears, can be constructed. $\xi$ is a gauge fixing parameter which will be set to $\xi=0$ in the following (Landau gauge). In total, eq. (2.2) has 5 running couplings $\left(Z_{\psi}, \bar{e}, Z_{a}, \tilde{\bar{g}}\right.$ and $\left.\bar{g}\right)$, which depend on the cutoff scale $k$. We are interested in their evolution in the infrared as we take $k \rightarrow 0$.

As mentioned in the Introduction above, we will here consider $\mathrm{QED}_{3}$ in the absence of a Chern-Simons term. Admitting the presence of the latter would in principle open up the possibility to investigate a number of other possible dualities $[4,5]$. Coupling the dynamical gauge field $a_{\mu}$ to a background field $A_{\mu}$ via $\frac{1}{2 \pi} \int \mathrm{d}^{3} x \epsilon^{\mu \nu \rho} a_{\mu} \partial_{\nu} A_{\rho}$, for instance, a duality to the Wilson-Fisher fixed-point of $\varphi^{4}$ theory has been conjectured recently $[4,5]$. In the future, it will be interesting to investigate this scenario within the present RG framework by adding the corresponding terms to the ansatz for the scale dependent effective action (2.2).

\section{$2.2 \beta$-functions}

In the simplest, point-like truncation for the couplings, projected onto the most singular point in frequency- and momentum space (the origin at $q=0$ ), the leading order $\beta$ functions for the gauge coupling $e^{2}$ and the two four-fermi couplings $\tilde{g}, g$ of eq. (2.2) are:

$$
\begin{aligned}
\partial_{t} e^{2}= & \left(\eta_{a}-1\right) e^{2} \\
\partial_{t} \tilde{g}= & \tilde{g}\left(1+2 \eta_{\psi}\right)-l_{\psi}^{1}\left(\frac{2 N_{\mathrm{f}}^{(4)}-1}{N_{\mathrm{f}}^{(4)}} \tilde{g}^{2}-\frac{3}{N_{\mathrm{f}}^{(4)}} \tilde{g} g-\frac{2}{N_{\mathrm{f}}^{(4)}} g^{2}\right) \\
& -l_{a, \psi}^{1,1}\left(2 \tilde{g} e^{2}+4 g e^{2}\right)+l_{a, \psi}^{2,1} 2 N_{\mathrm{f}}^{(4)} e^{4} \\
\partial_{t} g= & g\left(1+2 \eta_{\psi}\right)+l_{\psi}^{1}\left(\frac{1}{N_{\mathrm{f}}^{(4)}} \tilde{g} g+\frac{2 N_{\mathrm{f}}^{(4)}+1}{3 N_{\mathrm{f}}^{(4)}} g^{2}\right)-\frac{l_{a, \psi}^{1,1}}{3}\left(4 \tilde{g} e^{2}-2 g e^{2}\right) .
\end{aligned}
$$

Here we have abbreviated the scale-derivative $\partial_{t}=k \partial_{k}$. The set of $\beta$-functions eq. (2.3) is closed by two anomalous dimensions making it 5 equations and 5 couplings to solve. $\eta_{\psi}$ for the electrons

$$
\eta_{\psi}=-\frac{e^{2}}{3}\left[2 \tilde{m}_{a, \psi}^{1,1}-2 m_{a, \psi}^{2,1}\right]
$$

turns out to be negative in the regimes of interest and the threshold coefficients here take the form

$$
\begin{aligned}
\tilde{m}_{a, \psi}^{1,1} & =\frac{3}{2}-\frac{1}{6} \eta_{\psi}-\frac{1}{4} \eta_{a} \\
m_{a, \psi}^{2,1} & =1-\frac{1}{4} \eta_{a} .
\end{aligned}
$$


The photon anomalous dimension $\eta_{a}$ is physically caused by decay and recombination into electron-positron pairs. It takes the form

$$
\begin{aligned}
\eta_{a}= & N_{\mathrm{f}}^{(4)} e^{2} \frac{1}{\zeta^{2}} \int_{0}^{\infty} \mathrm{d} y\left\{\frac{2}{3} \frac{\partial_{t} r_{\psi}(y)-\eta_{\psi} r_{\psi}(y)}{\sqrt{y}\left[1+r_{\psi}(y)\right]^{3}}-\frac{1}{2} \int_{-1}^{1} \mathrm{~d} x \frac{\sqrt{y} x^{2}-\zeta x}{y-2 \zeta x \sqrt{y}+\zeta^{2}}\right. \\
& {\left.\left[\frac{\left[\partial_{t} r_{\psi}\right](y)-\eta_{\psi} r_{\psi}(y)}{\left[1+r_{\psi}(y)\right]^{2}\left[1+r_{\psi}\left(y-2 \zeta x \sqrt{y}+\zeta^{2}\right)\right]}+\frac{\left[\partial_{t} r_{\psi}\right]\left(y-2 \zeta x \sqrt{y}+\zeta^{2}\right)-\eta_{\psi} r_{\psi}\left(y-2 \zeta x \sqrt{y}+\zeta^{2}\right)}{\left[1+r_{\psi}(y)\right]\left[1+r_{\psi}\left(y-2 \zeta x \sqrt{y}+\zeta^{2}\right)\right]^{2}}\right]\right\} }
\end{aligned}
$$

which has a finite $\zeta \rightarrow 0$ limit. We also absorbed explicit $k$-dependences into renormalized gauge and induced four-fermion couplings

$$
\tilde{g} \equiv \frac{\tilde{\bar{g}} k}{Z_{\psi}^{2}}, \quad g \equiv \frac{\bar{g} k}{Z_{\psi}^{2}}, \quad e^{2} \equiv \frac{\bar{e}^{2}}{Z_{\psi}^{2} Z_{a} k} .
$$

The threshold functions appearing in eq. (2.3) are (for the linear Litim regulator)

$$
\begin{aligned}
l_{\psi}^{1} & =\frac{2}{3}-\frac{1}{6} \eta_{\psi} \\
l_{a, \psi}^{1,1} & =\frac{4}{3}-\frac{1}{6} \eta_{\psi}-\frac{2}{15} \eta_{a} \\
l_{a, \psi}^{2,1} & =2-\frac{1}{6} \eta_{\psi}-\frac{4}{15} \eta_{a}
\end{aligned}
$$

and are positive in the regimes of interest, that is, the "RG-corrections" by the anomalous dimensions are subdominant when compared to the leading term.

The $5 \beta$-functions eq. (2.3) and eqs. (2.4), (2.6) have scale-invariant, real-valued solutions for large enough $N_{\mathrm{f}}^{(4)}>N_{\mathrm{f}, \mathrm{c}}^{(4)}$; these signify a conformal phase. We now first describe the nature of these conformal fixed-points and subsequently explain how the scaling breaks down at $N_{\mathrm{f}}^{(4)}=N_{\mathrm{f}, \mathrm{c}}^{(4)}$.

\subsection{Recap of interacting conformal fixed point for $N_{\mathrm{f}}^{(4)}>N_{\mathrm{f}, \mathrm{c}}^{(4)}$}

Due to charge conservation, the photon anomalous dimension is exactly equal to one for any value of $N_{f}^{(4)}>N_{\mathrm{f}, \mathrm{c}}^{(4)}$ :

$$
\eta_{*}^{a}=1
$$

that is, along the line of interacting conformal fixed points corresponding to the conformal phase. This follows from eq. (2.3a). Since $\eta_{*}^{a}$ depends on $e_{*}^{2}$ itself, this fixes the numerical value of the gauge coupling, given in figure 1 , as a function of $N_{\mathrm{f}}^{(4)}$. The values of $\eta_{\psi}^{*}$ depend on the number of fermion flavors. This follows from a solution of the coupled equations for the anomalous dimensions eqs. (2.4), (2.6). Its values are given in figure 1 for the linear regulator and $\zeta \rightarrow 0$. Alternative techniques to access the conformal phase and its exponents and operator dimensions are the $1 / N_{\mathrm{f}}$ expansion, which offers perturbative control for sufficiently large $N_{\mathrm{f}}$ (e.g.: $[9,15-21]$ ), and the $\epsilon$-expansion around $d=4$ in the limit $\epsilon \rightarrow 1$ (e.g.: $[9,10,14]$ ). 


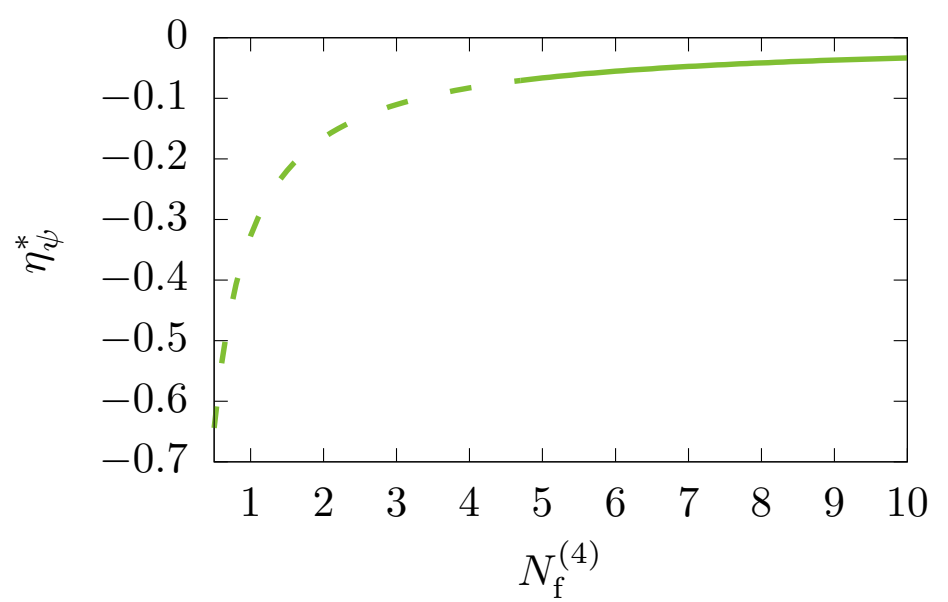

\begin{tabular}{|c|c|c|}
\hline$N_{\mathrm{f}}^{(4)}$ & $\eta_{\psi}^{*}$ & $e_{*}^{2}$ \\
\hline 5 & -0.066 & 0.195 \\
6 & -0.055 & 0.163 \\
7 & -0.048 & 0.140 \\
8 & -0.042 & 0.123 \\
9 & -0.037 & 0.109 \\
10 & -0.033 & 0.099 \\
\hline
\end{tabular}

Figure 1. Fermion anomalous dimension and value of the gauge coupling in the conformal phase as a function of the flavor number $N_{\mathrm{f}}^{(4)}$. The dashed line indicates the regime below $N_{\mathrm{f}, \mathrm{c}}^{(4)}$, where the conformal fixed point becomes unstable and likely spontaneous symmetry breaking sets in.

It is a feature of the $\beta$-functions that the flow of the gauge coupling (2.3a) and consequently the universal fixed-point values $e_{*}^{2}\left(N_{\mathrm{f}}\right)$ shown in figure 1 do not depend on $\tilde{g}$ or $g$. At the level of the perturbative Ward identity, this is diagrammatically due to Furry's theorem, i.e., the vanishing of graphs with an odd number of external gauge field insertions. This decoupling of the gauge flow from the fermion sector permits a simplified analysis of symmetry breaking patterns. $e^{2}$ may be viewed as an external parameter for the fermionic flow equations (2.3b) and (2.3c). Here we have taken the fermionic couplings to be not fundamental in the UV, $\tilde{g}_{k=\Lambda}=g_{k=\Lambda}=0$. They first need to be generated by gauge field fluctuations.

We now recapitulate how to detect symmetry breaking from the flows of eqs. (2.3), (2.4), (2.6).

\subsection{Breakdown criterion of conformal scaling at $N_{\mathrm{f}, \mathrm{c}}^{(4)}$}

For sufficiently large $N_{\mathrm{f}}^{(4)}>N_{\mathrm{f}, \mathrm{c}}^{(4)}$, the initial values, $g_{k=\Lambda}=\tilde{g}_{k=\Lambda}=0$ lie in the basin of attraction of a conformal fixed point. Note that in general there are four fixed point solutions, only one of which is infrared attractive. This is the conformal fixed-point at finite $e_{k \rightarrow 0}^{2}=e_{*}^{2}$ (labelled as $\mathcal{O}$ ).

For $N_{\mathrm{f}}^{(4)} \leq N_{\mathrm{f}, \mathrm{c}}^{(4)}$, however, the four-fermi couplings at $\mathcal{O}$ start developing imaginary parts, which is indicative of spontaneous symmetry breaking and the phase boundary between the conformal phase and a phase with spontaneously broken symmetry.

In figure 2, we plot the fixed-point values of $g$ and $\tilde{g}$ in the complex plane for varying $N_{\mathrm{f}}^{(4)}$. We observe that at $N_{\mathrm{f}}^{(4)} \leq N_{\mathrm{f}, \mathrm{c}}^{(4)}=4.7$ the couplings develop imaginary parts. This estimate is coincidentally close to a recent computation from the F-theorem and a resummed $\epsilon$-expansion $N_{\mathrm{f}, \mathrm{c}}^{(4)} \approx 4.4$ [10] and another recent estimate from the $\epsilon$-expansion at $N_{\mathrm{f}, \mathrm{c}}^{(4)} \approx 4.5[14]$. 


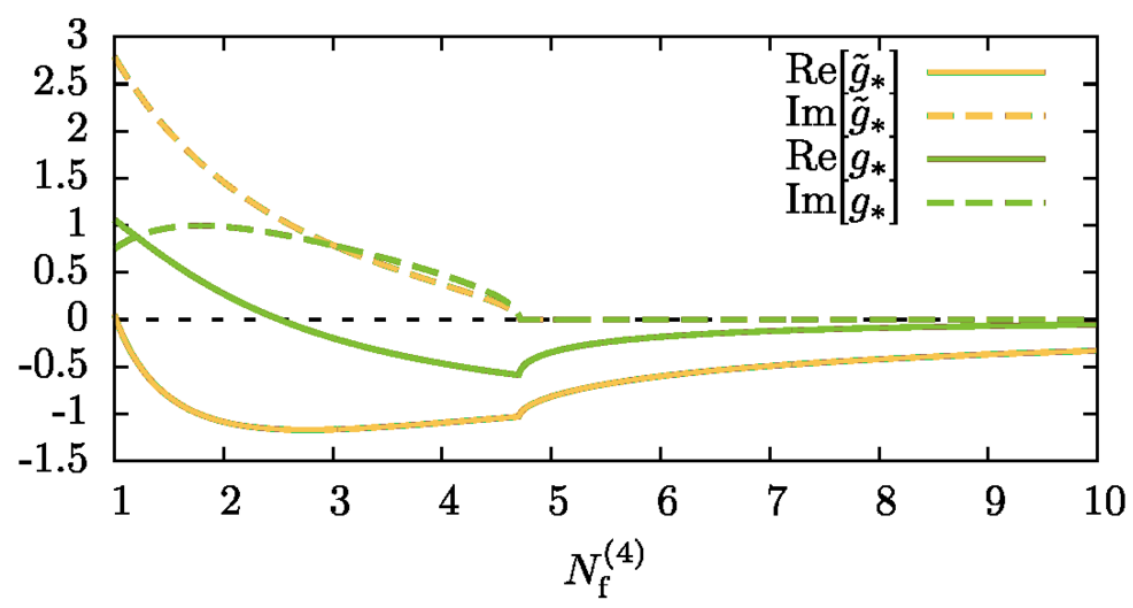

Figure 2. Fixed point values of the fermionic couplings $\tilde{g}$ and $g$ at the scale-invariant/conformal fixed point $\mathcal{O}$ for a range of $N_{\mathrm{f}}^{(4)}>N_{\mathrm{f}, \mathrm{c}}^{(4)}$. The gauge coupling is set to the respective fixed point value $e_{*}^{2}\left(N_{\mathrm{f}}^{(4)}\right)$. Finite imaginary parts indicate spontaneous symmetry-breaking. Here this happens below $N_{\mathrm{f}, \mathrm{c}}^{(4)}=4.7$.

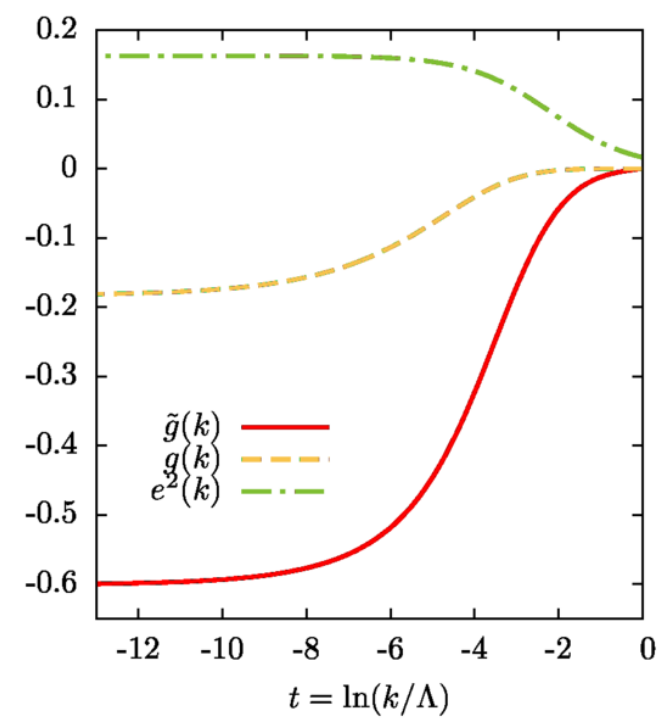

(a) $N_{\mathrm{f}}^{(4)}=6>N_{\mathrm{f}, \mathrm{c}}^{(4)}$.

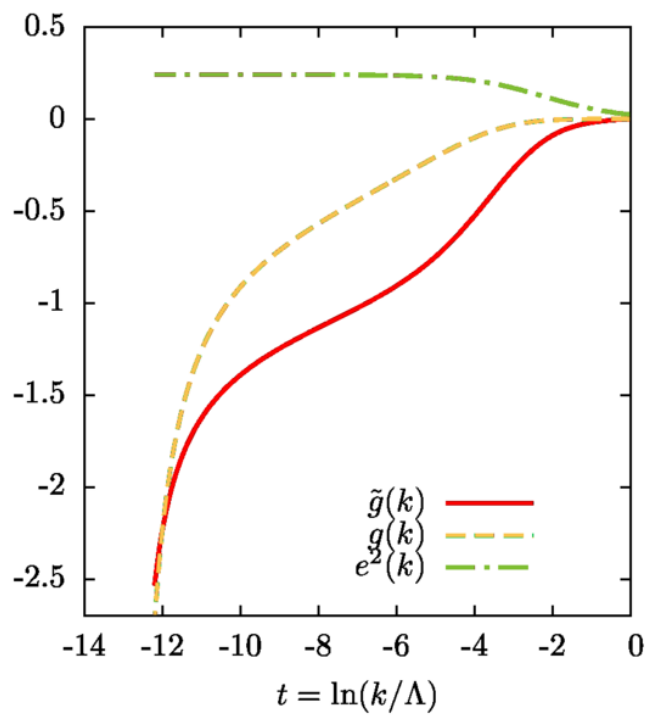

(b) $N_{\mathrm{f}}^{(4)}=4<N_{\mathrm{f}, \mathrm{c}}^{(4)}$.

Figure 3. Explicit solutions of the flow equations in the conformal phase (a) and in the phase with putatively broken symmetry (b). In the second case, the fermionic flow diverges at $t_{\mathrm{sb}}=-12.9$.

As shown in figure 3, an explicit solution of the 5 coupled flow equations as a function of $k$ confirms this picture: for $N_{\mathrm{f}}^{(4)} \leq 4.7$, the four-fermi couplings diverge at some finite scale $k_{\mathrm{sb}}$. These runaway flows indicate that fluctuations in one, or several, fermion bilinear channels become so strong that one, or a combination, of bilinears are likely to condense and spontaneously break the conformal symmetry. 


\section{Dual $\mathrm{QED}_{3}$ with a single 2-component fermion $N_{\mathrm{f}}^{(2)}=1$}

For 2D surfaces of certain 3D topological insulators and possibly other topological phases, we are interested in $\mathrm{QED}_{3}$ with a single two-component (composite) fermion coupled to an emergent $\mathrm{U}(1)$ gauge field. Therefore, the 4D representation for the fermionic spinors is not applicable and we need to move to the $2 \mathrm{D}$-representation and set $N_{\mathrm{f}}^{(2)}=1$.

To that end, consider the two linearly independent fermionic interactions in the $2 \mathrm{D}$ representation defined in the appendix of ref. [8]:

$$
\left(\bar{\psi}^{a} \gamma_{45} \psi^{a}\right)_{a=1 \ldots N_{\mathrm{f}}^{(4)}}^{2} \rightarrow\left(\bar{\chi}^{i} \chi^{i}\right)_{i=1 \ldots N_{\mathrm{f}}^{(2)}}^{2}, \quad\left(\bar{\psi}^{a} \gamma_{\mu} \psi^{a}\right)_{a=1 \ldots N_{\mathrm{f}}^{(4)}}^{2} \rightarrow\left(\bar{\chi}^{i} \sigma_{\mu} \chi^{i}\right)_{i=1 \ldots N_{\mathrm{f}}^{(2)}}^{2}
$$

In the special case of $N_{\mathrm{f}}^{(2)}=1$, it is

$$
-3(\bar{\chi} \chi)^{2}=\left(\bar{\chi} \sigma_{\mu} \chi\right)^{2} .
$$

There is only one independent fermionic interaction term left. The ansatz for the effective action (2.2) then reduces to

$$
\Gamma_{k}[\bar{\psi}, \psi, a]=\int \mathrm{d}^{3} x\left\{\bar{\chi}\left(i Z_{\psi} \not \partial+\bar{e} \not \phi\right) \chi+\frac{Z_{a}}{4} F_{\mu \nu} F_{\mu \nu}+\frac{Z_{a}}{2 \xi}\left(\partial_{\mu} a_{\mu}\right)^{2}+\bar{\lambda}(\bar{\chi} \chi)^{2}\right\}
$$

with $\bar{\lambda}=\tilde{\bar{g}}_{k}-3 \bar{g}_{k}$. Making use of the flow equations (2.3), the $\beta$-function for $\bar{\lambda}$ can be obtained from $\partial_{t} \bar{\lambda}=\partial_{t} \tilde{\bar{g}}_{k}-3 \partial_{t} \bar{g}_{k}$. Consequently, the flow equation for the dimensionless renormalized coupling $\lambda=\bar{\lambda} k Z_{\psi}^{-2}$ is given by

$$
\partial_{t} \lambda=\lambda\left(1+2 \eta_{\psi}\right)+2 l_{a, \psi}^{1,1} \lambda e^{2}+l_{a, \psi}^{2,1} e^{4} .
$$

The key feature of this equation is the absence of a $\lambda^{2}$ term. This is due to cancellations in the $\beta$-function special to the $N_{\mathrm{f}}^{(2)}=1$ case as we explain in appendix B. This structural specialty is already visible upon setting $N_{\mathrm{f}}^{(4)}=1 / 2$ in eq. (2.3b); then the $\tilde{g}^{2}$ in that equation disappears and the fixed-point structure qualitatively changes. Further implementing the symmetry eq. (3.2), then yields eq. (3.4) for a single four-fermi coupling. Of course, this $\beta$-function eq. (3.4) may also be derived directly from applying the Polchinski-Wetterich equation $[22,23]$ to the ansatz (3.3). This is performed explicitly in appendix A.

\subsection{Interacting conformal fixed point}

We now adapt the symmetry-breaking analysis explained above to a reduced number of equations, feeding the gauge sector and anomalous dimensions into eq. (3.4). Given the IR fixed point of the gauge sector $\left(e^{2}=e_{*}^{2}=1.59, \eta_{a}^{*}=1, \eta_{\psi}^{*}=-0.64\right)$ as an input, and because $\partial_{t} \lambda$ is linear in $\lambda$, there can be only one fixed point solution to eq. (3.4):

$$
\lambda_{*}=-\frac{l_{A, \psi}^{2,1} e_{*}^{4}}{1+2 \eta_{\psi}+2 l_{A, \psi}^{1,1} e_{*}^{2}}=-1.20,\left.\quad \frac{\partial\left(\partial_{t} \lambda\right)}{\partial \lambda}\right|_{\left(\lambda_{*}\right)}=1+2 \eta_{\psi}+l_{A, \psi}^{1,1} e_{*}^{2}=3.87
$$

The numerical values are provided for the linear regulator at $\zeta=0$ and in Landau gauge as before. Given the positive slope of $\partial_{t} \lambda$, the fixed point $\lambda_{*}$ is found to be infrared attractive. Therefore, no runaway flow occurs for arbitrary initial values $e_{k=\Lambda}^{2}$ and the fixed-point structure and explicit flows (see figure 4) preserve scaling/conformal invariance as $k \rightarrow 0$. 


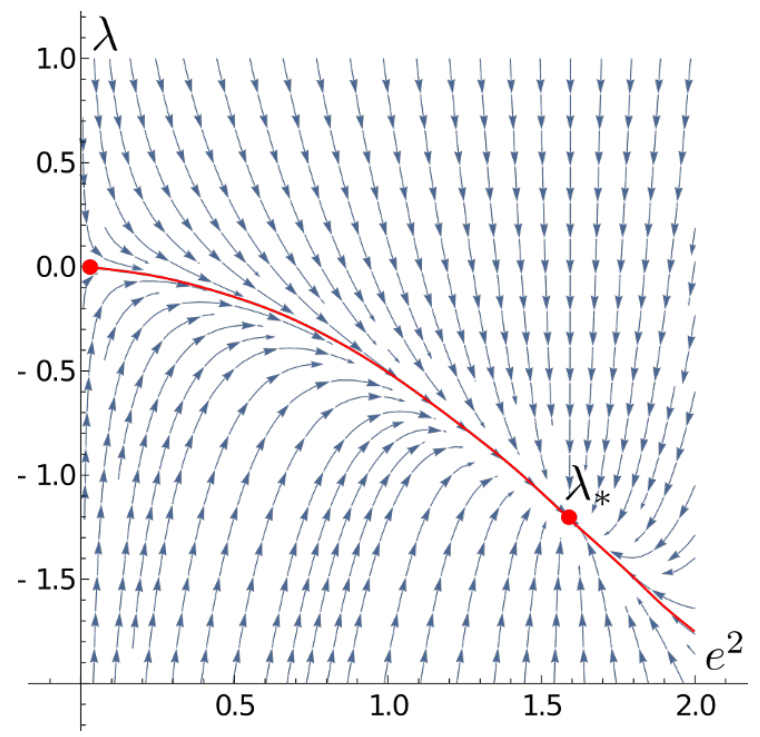

(a)

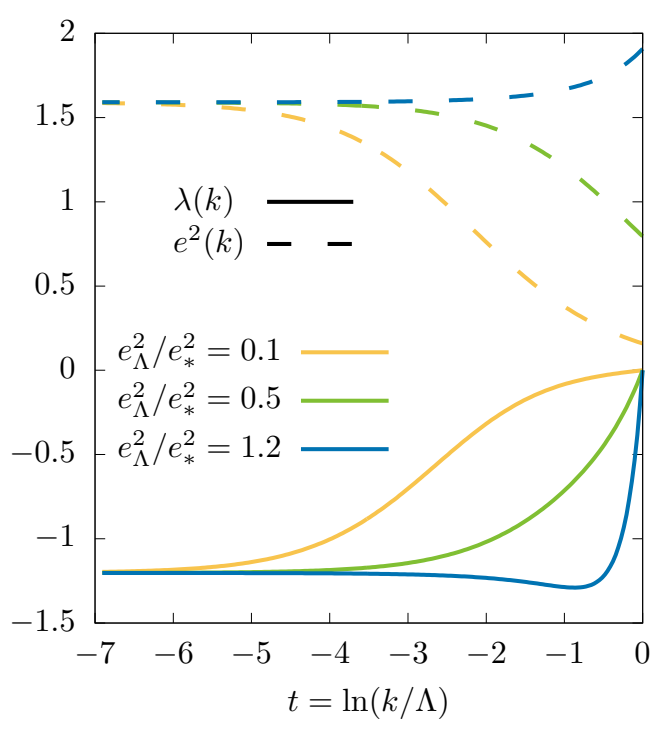

(b)

Figure 4. (a): universal location of the interacting conformal fixed point in the plane of interaction couplings for $N_{\mathrm{f}}^{(2)}=1$. Arrows point towards the infrared. (b): explicit numerical solutions for a range of initial values $e_{\Lambda}^{2}$ showing convergence of both, the gauge coupling $e^{2}$ and the four-fermi coupling $\lambda$, toward fixed-point plateaus in the infrared as $k \rightarrow 0$.

\subsection{Discussion}

In principle, by virtue of eqs. (3.1), (3.2) a runaway flow in the four-fermi sector with gauge-invariant regularization can lead to quadratic mass terms $\sim m \bar{\chi} \chi$ and spontaneous background currents $\sim\left\langle j^{\mu}\right\rangle \bar{\chi} \sigma^{\mu} \chi$ thereby breaking time-reversal and space-reflection symmetry. In fact, this parity anomaly appears generically if the entire large group of gauge transformations is allowed and Chern-Simons terms are induced [24-27]. But eq. (2.1) with $N_{\mathrm{f}}^{(2)}=1$ is an effective dual theory for the $2 \mathrm{D}$ surface of certain topological insulators. Then $\chi$ is actually a composite fermion field, which is electrically neutral and the standard large gauge transformations need to be modified such that no Chern-Simons term from a parity anomaly is allowed [2].

We note that a possible "strong form" of a Dirac fermion duality would relate eq. (2.1) with $N_{\mathrm{f}}^{(2)}=1$ to the partition function of a single, non-interacting Dirac cone [7]. In this scenario, it can be asserted that if $m_{e}=0$ for the free Dirac cone, than $m=0$ for the composite fermions of dual $\mathrm{QED}_{3}$. Our computation is in line with this reasoning.

\section{Implications of a possible "strong" Dirac fermion duality}

In this section, and based on the considerations above, we want to take for granted this strong duality on the level of the path integral between eq. (1.1) and a free Dirac cone of electrically charged fermions $\bar{\psi}_{e}, \psi_{e}$ [7]. For a given physical electromagnetic field $A_{\mu}$ coupling the electric charge, we have that

$$
\mathcal{L}_{\text {free }}=\bar{\psi}_{e}\left[i \gamma^{\mu}\left(\partial_{\mu}-i e_{\mathrm{phys}} A_{\mu}\right)\right] \psi_{e},
$$


is "dual" to the theory

$$
\mathcal{L}_{\mathrm{cf}}=\bar{\psi}\left[i \gamma^{\mu}\left(\partial_{\mu}-i g_{\mathrm{cf}} a_{\mu}\right)\right] \psi+\frac{g_{\mathrm{cf}} e_{\mathrm{phys}}}{4 \pi} \epsilon^{\mu \nu \lambda} A_{\mu} \partial_{\nu} a_{\lambda} .
$$

The duality then implies that both eq. (1.1) and eq. (4.1) describe the same underlying physical system and the response to actual physical electromagnetic fields must be the same. In eq. (4.1), the physical electromagnetic field couples, as usual, to the electronic current, whereas in eq. (4.2) $A_{\mu}$ couples to the gauge flux of the emergent photon.

We now survey two aspects of this duality: constraints for the topological current correlator in subsection 4.1 and operator dimensions in subsection 4.2.

\subsection{Constraining the topological current correlator}

The current-current correlator on the free Dirac cone side of the duality eq. (4.1) is

$$
\left\langle j_{\mu}(-p) j_{\nu}(p)\right\rangle=-N_{\mathrm{f}}^{(2)} C_{j}^{\text {free }}|p|\left(\delta_{\mu \nu}-\frac{p_{\mu} p_{\nu}}{p^{2}}\right)
$$

where $j_{\mu}=\bar{\psi}_{e} \gamma_{\mu} \psi_{e}$ is the physical current dual to the physical electromagnetic gauge field $A_{\mu}, N_{\mathrm{f}}^{(2)}=1$ for one single two-component fermion, and

$$
C_{j}^{\text {free }}=\frac{e_{\text {phys }}^{2}}{16} .
$$

This equation is exact; there are no interaction corrections.

On the composite fermion (cf) side, the physical electromagnetic gauge field couples (via a $A_{\mu} J_{\mu}^{\text {top }}$ term in the Lagrangian) to the topological current via

$$
J_{\mu}^{\mathrm{top}}=\frac{g_{\mathrm{cf}}}{4 \pi} \epsilon_{\mu}^{\nu \kappa} \partial_{\nu} a_{\kappa} .
$$

Since both sides of the duality should describe the same physical reality, we should have

$$
\left\langle j_{\mu}(-p) j_{\nu}(p)\right\rangle_{\text {free }}=\left\langle J_{\mu}^{\mathrm{top}}(-p) J_{\nu}^{\mathrm{top}}(p)\right\rangle_{\mathrm{cf}}
$$

Now, for zero doping and in a conformal phase with conserved topological current $\partial_{\mu} J_{\mu}^{\text {top }}=0$, the correlator must also have the form of eq. (4.3) but with an independent universal constant

$$
\left\langle J_{\mu}^{\mathrm{top}}(-p) J_{\nu}^{\mathrm{top}}(p)\right\rangle=-N_{\mathrm{cf}}^{(2)} C_{J}^{\mathrm{top}}|p|\left(\delta_{\mu \nu}-\frac{p_{\mu} p_{\nu}}{p^{2}}\right)
$$

with $N_{\mathrm{cf}}^{(2)}=1$. However, the number $C_{J}^{\text {top }}$ is not known, since the composite fermion theory is interacting. We can now use eq. (4.6) to determine $C_{J}^{\text {top }}$ exactly thus constraining perturbative computations for $C_{J}^{\text {top }}$. The best estimate for $C_{J}^{\text {top }}$ is in eq. (4.3.) of ref. [9], abbreviated as GTK:

$$
\begin{aligned}
\left\langle J_{\mu}^{\mathrm{top}, \mathrm{GTK}}(-p) J_{\nu}^{\mathrm{top}, \mathrm{GTK}}(p)\right\rangle= & -\frac{8|p|}{\pi^{2} N_{G T K}}\left(1+\frac{1}{N_{G T K}}\left(8-\frac{736}{9 \pi^{2}}\right)+O\left(1 / N_{G T K}^{2}\right)\right)\left(\delta_{\mu \nu}-\frac{p_{\mu} p_{\nu}}{p^{2}}\right) \\
\equiv & -|p|\left[\frac{8}{\pi^{2} N_{G T K}}\left(1+\frac{1}{N_{G T K}}\left(8-\frac{736}{9 \pi^{2}}\right)\right)+\Delta X^{\mathrm{top}}\left(N_{G T K}\right)\right] \\
& \times\left(\delta_{\mu \nu}-\frac{p_{\mu} p_{\nu}}{p^{2}}\right),
\end{aligned}
$$


where in the second line we have subsumed the unknown interaction corrections to all orders in $1 / N_{G T K}$ into the variable $\Delta X^{\text {top }}\left(N_{G T K}\right)$. After matching conventions

$$
\begin{aligned}
N_{G T K} & =2 N_{\mathrm{f}}^{(2)} \\
J_{\mu}^{\mathrm{top}, \mathrm{GTK}} & =2 J_{\mu}^{\mathrm{top}}
\end{aligned}
$$

and invoking eqs. (4.4), (4.6), we obtain for $N_{\mathrm{f}}^{(2)}=1$ (in units where coupling constants are unity)

$$
\begin{aligned}
\left\langle J_{\mu}^{\mathrm{top}, \mathrm{GTK}}(-p) J_{\nu}^{\mathrm{top}, \mathrm{GTK}}(p)\right\rangle & =-|p|\left[0.347365+\Delta X^{\mathrm{top}}\left(N_{G T K}=2\right)\right]\left(\delta_{\mu \nu}-\frac{p_{\mu} p_{\nu}}{p^{2}}\right) \\
\Delta X^{\mathrm{top}}\left(N_{G T K}=2\right) & =-0.0973652 .
\end{aligned}
$$

Knowledge of the exact value for this and other universal constants may help to constrain perturbative computations of thermodynamics, entanglement, and response functions of interacting, dual $\mathrm{QED}_{3}$ and possibly extensions thereof.

\subsection{Scaling dimension of composite mass operator at one-loop}

Here we compute the singular corrections to the scaling dimension of the composite mass operator $m=\bar{\psi} \psi$ for the fermion fields at the fixed-point of subsection 3.1:

$$
\int d^{3} x m Z_{m} Z_{\psi} \bar{\psi} \psi
$$

Defining the anomalous exponents ( $\Lambda$ is the running cutoff scale),

$$
\eta_{\psi}=-\frac{\partial \log Z_{\psi}}{\partial \log \Lambda}, \quad \eta_{m}=-\frac{\partial \log Z_{m}}{\partial \log \Lambda},
$$

the total correction to the scaling dimension of the mass operator is then

$$
\eta_{\text {mass }}=\eta_{m}+\eta_{\psi}
$$

We first compute the fermionic field renormalization $Z_{\psi}$ and $\eta_{\psi}$ from the one-loop selfenergy shown in figure 5 . The photon anomalous dimension is $\eta_{a}=1$ and we can use the standard overdamped one-loop form for $N_{\mathrm{f}}^{(2)}=1$ :

$$
D_{\mu \nu}(q)=\frac{16}{g_{\mathrm{cf}}^{2}|q|}\left(\eta_{\mu \nu}-(1-\xi) \frac{q_{\mu} q_{\nu}}{q^{2}}\right)
$$

where $g_{\mathrm{cf}}$ is the photon-fermion coupling as denoted in eq. (4.2). We may use Feynman gauge $\xi=1$ in what follows. With this, the singular fermion self-energy correction is:

$$
\begin{aligned}
\delta \Sigma_{\psi}(q) & =g_{\mathrm{cf}}^{2} \int \frac{d^{3} p}{(2 \pi)^{3}}\left(-\gamma^{\mu}\right) G_{\psi}(k+q)\left(-\gamma^{\nu}\right) D_{\mu \nu}(q) \\
& =-\frac{8}{3 \pi^{2}} \not k \log \left(\frac{\Lambda}{|k|}\right) .
\end{aligned}
$$




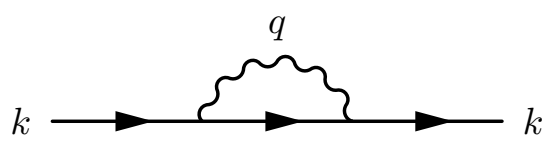

Figure 5. One-loop fermion self energy for $Z_{\psi}$ and the corresponding anomalous dimension $\eta_{\psi}$.

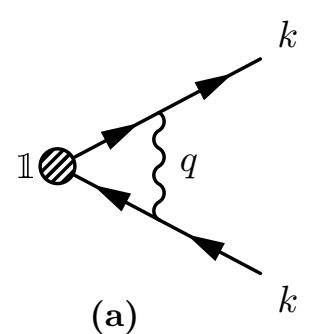

(a)

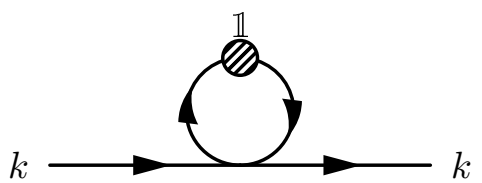

(b)

Figure 6. One-loop graphs for insertions of the mass operator $m=\bar{\psi} \psi$. (a) is computed in the text. (b) arises from the four-fermi coupling $\lambda$ and does not produce singular corrections, when approximating $\lambda$ as momentum-independent. Upon keeping the momentum-dependence of the fourfermi coupling, for example via $\sigma$-meson exchange [29], (b) can also generate singular corrections to operator dimensions.

Invoking the usual RG improvement, the anomalous dimension for the electrons obtains as

$$
\eta_{\psi}=\frac{8}{3 \pi^{2}}
$$

in agreement with a previous calculation upon setting their $N$ to $1 / 2$ and gauge fixing $\xi=1[28]$.

The one-loop graphs for the correction to the mass operator $\delta m(k)$ are shown in figure 6. Graph (a) is non-vanishing and yields the divergent correction

$$
\delta m(k)=\mathbb{1} \frac{24}{\pi^{2}} \log \left(\frac{\Lambda}{|k|}\right), \quad \eta_{m}=-\frac{24}{\pi^{2}} .
$$

Adding the exponents as per eq. (4.13) we get the final result

$$
\eta_{\text {mass }}=-\frac{64}{3 \pi^{2}}
$$

which agrees with the leading term in a computation of the same quantity by Gracey upon setting his $N_{f}=1 / 2[30]$.

A strong form Dirac fermion duality would require $\eta_{\text {mass }}=0$ [7]. Implementing the momentum-dependent four-fermi coupling via $\sigma$-meson exchange [29] will produce corrections to eq. (4.18), as may other additional terms to the truncation eq. (3.3). It is an interesting project to establish explicitly the exponent identities conjectured by the duality.

We checked that for the insertion of the (conserved) current operator, $\int d^{3} x j_{\mu} Z_{j_{\mu}} Z_{\psi}$ $\bar{\psi}_{\mathrm{cf}} \gamma^{\mu} \psi_{\mathrm{cf}}$, with $j_{\mu}=\bar{\psi}_{\mathrm{cf}} \gamma_{\mu} \psi_{\mathrm{cf}}$, these cancellations appear explicitly.

$$
\eta_{\text {current }}=\eta_{j_{\mu}}+\eta_{\psi}=-\frac{8}{3 \pi^{2}}+\frac{8}{3 \pi^{2}}=0
$$




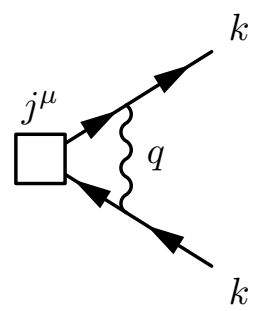

Figure 7. One-loop correction to insertions of the current operator $j_{\mu}=\bar{\psi}_{\mathrm{cf}} \gamma_{\mu} \psi_{\mathrm{cf}}$.

Evaluating figure 7 results in

$$
\delta j^{\mu}(k)=\frac{8}{3 \pi^{2}}\left(\gamma^{\mu} \log \left(\frac{\Lambda}{|k|}\right)-\frac{k^{\mu}}{k^{2}} \not k\right),
$$

and produces a singular correction with opposite sign $\eta_{j_{\mu}}=-\eta_{\psi}$ ensuring the cancellation eq. (4.19).

\section{Conclusions}

This paper explored aspects of a particle-vortex duality for Dirac fermions in two space dimensions. We raised the question whether a single two-component Dirac fermion coupled to $\mathrm{U}(1)$ gauge field can have a conformally invariant ground state and found indications that this may be the case, at least for the initially only weakly coupled model. The CFT we found is not free but has non-trivial anomalous dimensions and finite four-fermi couplings. It would be desirable to have a (non-perturbative) proof of the absence of spontaneous symmetry-breaking in the ground state of eq. (1.1) by generalizing the Vafa-Witten theorem to smaller flavor numbers down to $N_{f}^{(2)}=1$.

A strong-form duality between dual $\mathrm{QED}_{3}$ and a free Dirac cone would constrain operator dimensions and, as we tried to show, also universal constants of electromagnetic response functions.

In the future, it will be interesting to establish exponent identities and possibly emergent conservation laws of dual $\mathrm{QED}_{3}$ more completely and to higher loop order. To strengthen the link to a specific condensed matter situation, a further topic of interest are the "non-universalities" of dual $\mathrm{QED}_{3}$ such as the kinematics, velocities, additional interactions, and energy scales below which the continuum field theories for the effective degrees of freedoms emerge.

\section{Acknowledgments}

We thank A. Altland, J. Braun, S. Diehl and A. Rosch for fruitful discussions. We also thank H. Gies, I. Klebanov and M. Metlitski for insightful correspondences. This work was supported by the Leibniz Prize of A. Rosch and by the German Research Foundation (DFG) through the Institutional Strategy of the University of Cologne within the German Excellence Initiative (ZUK 81). 


\section{A Derivation of $\beta$-function $\partial_{t} \lambda$ for four-fermi coupling $\lambda$}

The beta function for the single fermionic coupling $\lambda$ can be derived without making reference to the flow equations (2.3). Here we employ the Polchinski-Wetterich equation [23]

$$
\partial_{t} \Gamma_{k}=\frac{1}{2} \mathrm{~S} \operatorname{Tr}\left[\frac{\partial_{t} R_{k}}{\Gamma_{k}^{(2)}+R_{k}}\right]=\frac{1}{2} \mathrm{~S} \operatorname{Tr}\left[\tilde{\partial}_{t} \mathcal{P}_{k}\right]+\frac{1}{2} \mathrm{~S} \operatorname{Tr}\left[\tilde{\partial}_{t} \sum_{n=1}^{\infty} \frac{(-1)^{n-1}}{n}\left(\mathcal{P}_{k}^{-1} \mathcal{F}_{k}\right)^{n}\right]
$$

to extract $\partial_{t} \lambda$ from the ansatz (3.3). The expansion of this equation on terms of propagator and fluctuation matrices $\Gamma_{k}^{(2)}+R_{k}=\mathcal{P}_{k}^{-1}+\mathcal{F}_{k}$ facilitates a projection onto the respective operator structures. The propagator matrix is given by

$$
\begin{aligned}
\mathcal{P}_{k}^{-1} & =\delta^{(3)}(p-q)\left(\begin{array}{ccc}
P_{a}^{\mu \nu} & 0 & 0 \\
0 & 0 & -P_{\chi} \\
0 & -P_{\chi}^{T} & 0
\end{array}\right), \quad P_{\chi}=q \cdot\left[Z_{\psi}\left(1+r_{\psi}\right)\right]^{-1}, \\
P_{a} & =\left[\delta_{\mu \nu} q^{2}-q_{\mu} q_{\nu}\right] \cdot\left[Z_{a} q^{2}\left(1+r_{a}\right)\right]^{-1} .
\end{aligned}
$$

Here, only terms $\sim(\bar{\chi} \chi)$ are of interest. Therefore, any explicit dependence of the fluctuation matrix on the gauge field $a_{\mu}$ can safely be dropped from the outset and we get

$$
\mathcal{F}_{k}=\left(\begin{array}{ccc}
0 & \bar{e} \bar{\chi}_{q-p} \sigma_{\mu} & -\bar{e} \chi_{p-q}^{T} \sigma_{\mu}^{T} \\
-\bar{e} \sigma_{\nu}^{T} \bar{\chi}_{q-p}^{T} & -2 \bar{\lambda} \int_{p_{1}} \bar{\chi}_{p_{1}}^{T} \bar{\chi}_{p-q+p_{1}} & 2 \bar{\lambda} \int_{p_{1}}\left[\bar{\chi}_{p_{1}}^{T} \chi_{p-q+p_{1}}^{T}-\left(\bar{\chi}_{p_{1}} \chi_{p-q+p_{1}}\right) \mathbb{1}\right] \\
\bar{e} \sigma_{\nu} \chi_{p-q} & 2 \bar{\lambda} \int_{p_{1}}\left[\left(\bar{\chi}_{p_{1}} \chi_{p-q+p_{1}}\right) \mathbb{1}+\chi_{p_{1}} \bar{\chi}_{p-q+p_{1}}\right] & -2 \bar{\lambda} \int_{p_{1}} \chi_{p_{1}} \chi_{p-q-p_{1}}^{T}
\end{array}\right)
$$

Projecting onto spatially constant fermion fields $\chi_{p}:=\chi \delta^{(3)}(p)$, the basic building block of the expansion (A.1) can be expressed as

$$
\left[\mathcal{P}_{k}^{-1} \mathcal{F}_{k}\right]_{\mu \nu}=\delta^{(3)}(p-q)\left(\begin{array}{ccc}
0 & P_{a}^{\mu \nu} \bar{e} \bar{\chi} \sigma_{\nu} & -P_{a}^{\mu \nu} \bar{e} \chi^{T} \sigma_{\nu}^{T} \\
-P_{\chi} \bar{e} \sigma_{\mu} \chi & -P_{\chi} 2 \bar{\lambda}[(\bar{\chi} \chi) \mathbb{1}+\chi \bar{\chi}] & P_{\chi} 2 \bar{\lambda} \chi \chi^{T} \\
P_{\chi}^{T} \bar{e} \sigma_{\nu}^{T} \bar{\chi}^{T} & P_{\chi}^{T} 2 \bar{\lambda} \bar{\chi}^{T} \bar{\chi} & -P_{\chi}^{T} 2 \bar{\lambda}\left[\bar{\chi}^{T} \chi^{T}-(\bar{\chi} \chi) \mathbb{1}\right]
\end{array}\right)
$$

There are three contributions to the flow of the bare coupling $\bar{\lambda}$ which can be depicted diagrammatically as in figure 8 below.

Here, double lines symbolize the (full) renormalized propagators in (A.2).

Fermionic self interaction. This contribution does not involve the gauge vertex. Consequently, only the lower right submatrix of eq. (A.4) is needed. Projecting onto $(\bar{\chi} \chi)^{2}$ gives

$$
\left.\partial_{t} \bar{\lambda}\right|_{\bar{\lambda}^{2}}(\bar{\chi} \chi)^{2}=\frac{1}{\Omega} \frac{1}{2} \frac{-1}{2} \operatorname{STr}\left[\left(\mathcal{P}_{k}^{-1} \mathcal{F}_{k}\right)_{\bar{\lambda}^{2}}^{2}\right]=0
$$

where $\Omega$ is the three-dimensional spacetime volume. Thus, tracelessness of the quadratic term in the expansion enforces linearity of the $\beta$-function. This finding is crucial for $\mathrm{QED}_{3}$ being conformal for $N_{\mathrm{f}}^{(2)}=1$ and it only occurs for this flavor number. Some details of why this is the case are given in appendix $\mathrm{B}$ below. 


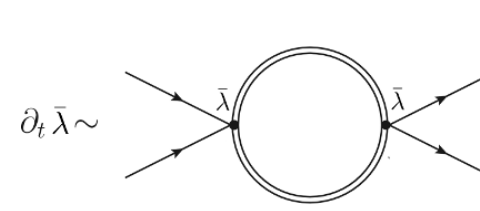

(a)

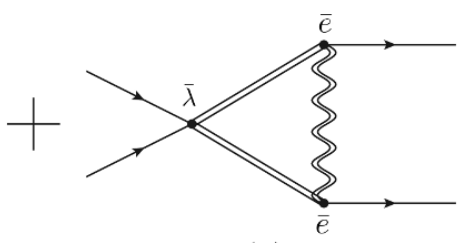

(b)

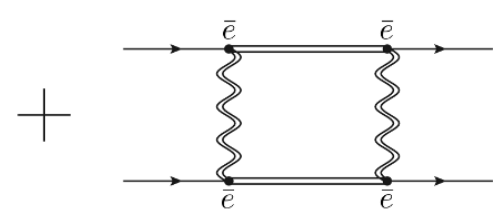

(c)

Figure 8. Diagrammatic representation of the contributions to $\partial_{t} \bar{\lambda}$. It is a special feature here that diagram (a) vanishes after the trace.

Triangle diagram. Both gauge and fermionic sectors are involved in the computation of this diagram. It is given by

$$
\left.\partial_{t} \bar{\lambda}\right|_{\bar{\lambda} \bar{e}^{2}}(\bar{\chi} \chi)^{2}=\frac{1}{\Omega} \frac{1}{2} \frac{1}{3} \mathrm{~S} \operatorname{Tr}\left[\left(\mathcal{P}_{k}^{-1} \mathcal{F}_{k}\right)_{\bar{\lambda} \bar{e}^{2}}^{3}\right]=\frac{l_{a, \psi}^{1,1}}{\pi^{2}} \frac{2}{k Z_{\psi}^{2} Z_{a}}(\bar{\chi} \chi)^{2}
$$

Here, the usual compact notation in terms of a threshold function has been introduced,

$$
l_{a, \psi}^{n_{a}, n_{\psi}}=k^{2 n_{A}-2 n_{\psi}-3} \int \mathrm{d} q q^{4}\left[n_{a} \frac{\partial_{t} r_{a}-\eta_{a} r_{a}}{P_{r_{a}}}+2 n_{\psi} \frac{1+r_{\psi}}{P_{r_{\psi}}}\left(\partial_{t} r_{\psi}-\eta_{\psi} r_{\psi}\right)\right] P_{r_{a}}^{-n_{a}} P_{r_{\psi}}^{-n_{\psi}}
$$

with

$$
P_{r_{a}}=q^{2}\left[1+r_{a}\right], \quad P_{r_{\psi}}=q^{2}\left[1+r_{\psi}\right]^{2} .
$$

Box diagram. The last diagram is particularly important as it is responsible to generate the fermionic interaction when starting from the $\mathrm{QED}_{3}$ action in the UV, where $\bar{\lambda}_{\Lambda}=0$. When computing its value, the lower right submatrix of eq. (A.4) may be ignored as only the gauge vertex contributes.

$$
\left.\partial_{t} \bar{\lambda}\right|_{\bar{e}^{4}}(\bar{\chi} \chi)^{2}=\frac{1}{\Omega} \frac{1}{2} \frac{-1}{4} \mathrm{~S} \operatorname{Tr}\left[\left(\mathcal{P}_{k}^{-1} \mathcal{F}_{k}\right)_{\bar{e}^{4}}^{4}\right]=\frac{l_{a, \psi}^{2,1}}{\pi^{2}} \frac{1}{k^{3} Z_{\psi}^{2} Z_{a}^{2}} \bar{e}^{4}(\bar{\chi} \chi)^{2}
$$

Renormalization and rescaling. In a last step, a renormalization of the couplings as in eq. (2.7) and the fields as $\chi \rightarrow \chi / \sqrt{Z_{\psi}}$ as well as a rescaling of $e^{2}$ and $\lambda$ with $\pi^{2}$ provides the final result for the $\beta$-function:

$$
\partial_{t} \lambda=\lambda\left(1+2 \eta_{\psi}\right)+\frac{k}{Z_{\psi}^{2}} \partial_{t} \bar{\lambda}=\lambda\left(1+2 \eta_{\psi}\right)+2 l_{A, \psi}^{1,1} \lambda e^{2}+l_{A, \psi}^{2,1} e^{4} .
$$

This reproduces eq. (3.4) of the main text.

\section{B Cancellation of $\lambda^{2}$ term from flavor trace in $\partial_{t} \lambda$}

In order to understand the origin of the cancellation enforcing linearity of the $\beta$-function (A.10) with respect to $\lambda$, it is necessary to revisit the corresponding contributions for general flavor number $N_{\mathrm{f}}^{(2)}$. Although there are two independent quartic fermion terms for $N_{\mathrm{f}}^{(2)}>1$ (see eq. (3.1)), it is sufficient to consider the generalized interaction

$$
(\bar{\chi} \chi)^{2} \rightarrow\left(\bar{\chi}^{i} \chi^{i}\right)_{i=1 \ldots N_{\mathrm{f}}^{(2)}}^{2}
$$


The fermion sector of the analog to eq. (A.4) is then given by

$$
\left[\mathcal{P}_{k}^{-1} \mathcal{F}_{k}\right]_{\bar{\chi} \chi}^{i j}=\delta^{(3)}(p-q) \frac{2 \bar{\lambda}}{N_{\mathrm{f}}^{(2)}}\left(\begin{array}{cc}
-P_{\chi}\left[(\bar{\chi} \chi) \mathbb{1} \delta^{i j}+\chi^{i} \chi^{j}\right] & P_{\chi} \chi^{i}\left(\chi^{j}\right)^{T} \\
P_{\chi}^{T}\left(\bar{\chi}^{i}\right)^{T} \bar{\chi}^{j} & -P_{\chi}^{T}\left[\left(\bar{\chi}^{i}\right)^{T}\left(\chi^{j}\right)^{T}-(\bar{\chi} \chi) \mathbb{1} \delta^{i j}\right]
\end{array}\right),
$$

where the flavor dependence of the fermion propagator has already been absorbed. It is then

$$
\begin{aligned}
\left.\partial_{t} \bar{\lambda}\right|_{\bar{\lambda}^{2}}\left(\bar{\chi}^{i} \chi^{i}\right)^{2} & =\frac{N_{\mathrm{f}}^{(2)}}{\Omega} \frac{1}{2}\left(\frac{-1}{2}\right) \operatorname{STr}\left[\left(\mathcal{P}_{k}^{-1} \mathcal{F}_{k}\right)_{\bar{\lambda}^{2}}^{2}\right]=2 \frac{l_{\psi}^{1}}{N_{\mathrm{f}}^{(2)} \pi^{2}} \operatorname{Tr}_{\mathrm{f}}\left[\left(\bar{\chi}^{a} \chi^{a}\right) \bar{\chi}^{i} \chi^{j}-\left(\bar{\chi}^{a} \chi^{a}\right)^{2} \delta^{i j}\right] \\
& =2 \frac{l_{\psi}^{1}}{N_{\mathrm{f}}^{(2)} \pi^{2}}\left(1-N_{\mathrm{f}}^{(2)}\right) \bar{\lambda}^{2}\left(\bar{\chi}^{i} \chi^{i}\right)^{2}
\end{aligned}
$$

where in the first line all traces but the flavor one have been performed and

$$
l_{\psi}^{n}=2 n k^{2 n-3} \int \mathrm{d} q q^{4}\left(\partial_{t} r_{\psi}-\eta_{\psi} r_{\psi}\right) \frac{1+r_{\psi}}{P_{r_{\psi}}^{n+1}} .
$$

We emphasize the prefactor in this expression vanishes for the case of interest $N_{\mathrm{f}}^{(2)}=1$ as announced in the main text. This is due to the absence of flavor off-diagonal terms in eq. (B.3) for this case. For $N_{\mathrm{f}}^{(2)}=1$, the flavor indices $a=i=j$ must fall onto each other and the bracket [...] in the first line of eq. (B.3) vanishes. Related vanishing of $\beta$-functions in the single-flavor case also appears in the Gross-Neveu model and in particular also holds at higher loop orders [11-13]. Complete cancellations of individual contractions in a $\beta$ function for a four-fermion vertex also appear in Luttinger liquids [31], although these are specific to spatial dimension $D=1$.

Open Access. This article is distributed under the terms of the Creative Commons Attribution License (CC-BY 4.0), which permits any use, distribution and reproduction in any medium, provided the original author(s) and source are credited.

\section{References}

[1] D.T. Son, Is the composite fermion a Dirac particle?, Phys. Rev. X 5 (2015) 031027 [arXiv: 1502.03446] [INSPIRE].

[2] M.A. Metlitski and A. Vishwanath, Particle-vortex duality of two-dimensional Dirac fermion from electric-magnetic duality of three-dimensional topological insulators, Phys. Rev. B 93 (2016) 245151 [arXiv: 1505.05142] [INSPIRE].

[3] C. Wang and T. Senthil, Dual dirac liquid on the surface of the electron topological insulator, Phys. Rev. X 5 (2015) 041031 [arXiv: 1505.05141] [INSPIRE].

[4] N. Seiberg, T. Senthil, C. Wang and E. Witten, A duality web in $2+1$ dimensions and condensed matter physics, Annals Phys. 374 (2016) 395 [arXiv: 1606.01989] [InSPIRE].

[5] A. Karch and D. Tong, Particle-vortex duality from 3d bosonization, Phys. Rev. X 6 (2016) 031043 [arXiv: 1606.01893] [INSPIRE]. 
[6] C. Vafa and E. Witten, Eigenvalue inequalities for fermions in gauge theories, Commun. Math. Phys. 95 (1984) 257 [inSPIRE].

[7] D.F. Mross, J. Alicea and O.I. Motrunich, Explicit derivation of duality between a free Dirac cone and quantum electrodynamics in (2+1) dimensions, Phys. Rev. Lett. 117 (2016) 016802 [arXiv: 1510.08455] [INSPIRE].

[8] J. Braun, H. Gies, L. Janssen and D. Roscher, Phase structure of many-flavor $Q E D_{3}$, Phys. Rev. D 90 (2014) 036002 [arXiv: 1404.1362] [INSPIRE].

[9] S. Giombi, G. Tarnopolsky and I.R. Klebanov, On $C_{J}$ and $C_{T}$ in conformal QED, JHEP 08 (2016) 156 [arXiv: 1602.01076] [INSPIRE].

[10] S. Giombi, I.R. Klebanov and G. Tarnopolsky, Conformal $Q E D_{d}, F$-theorem and the $\epsilon$ expansion, J. Phys. A 49 (2016) 135403 [arXiv: 1508.06354] [inSPIRE].

[11] W. Wetzel, Two loop $\beta$-function for the Gross-Neveu model, Phys. Lett. B 153 (1985) 297 [INSPIRE].

[12] J.A. Gracey, Three loop calculations in the O(N) Gross-Neveu model, Nucl. Phys. B 341 (1990) 403 [INSPIRE].

[13] C. Luperini and P. Rossi, Three loop $\beta$-function(s) and effective potential in the Gross-Neveu model, Annals Phys. 212 (1991) 371 [inSPIRE].

[14] L. Di Pietro, Z. Komargodski, I. Shamir and E. Stamou, Quantum electrodynamics in D $=3$ from the $\epsilon$ expansion, Phys. Rev. Lett. 116 (2016) 131601 [arXiv:1508.06278] [INSPIRE].

[15] J.A. Gracey, Computation of critical exponent $\eta$ at $o\left(1 / n_{f}^{2}\right)$ in quantum electrodynamics in arbitrary dimensions, Nucl. Phys. B 414 (1994) 614 [hep-th/9312055] [INSPIRE].

[16] W. Chen, M.P.A. Fisher and Y.-S. Wu, Mott transition in an anyon gas, Phys. Rev. B 48 (1993) 13749 [cond-mat/9301037] [INSPIRE].

[17] W. Rantner and X.-G. Wen, Spin correlations in the algebraic spin liquid: Implications for high-Tc superconductors, Phys. Rev. B 66 (2002) 144501 [INSPIRE].

[18] R.K. Kaul and S. Sachdev, Quantum criticality of U(1) gauge theories with fermionic and bosonic matter in two spatial dimensions, Phys. Rev. B 77 (2008) 155105 [arXiv:0801.0723] [INSPIRE].

[19] S.S. Pufu, Anomalous dimensions of monopole operators in three-dimensional quantum electrodynamics, Phys. Rev. D 89 (2014) 065016 [arXiv:1303.6125] [INSPIRE].

[20] Y. Huh and P. Strack, Stress tensor and current correlators of interacting conformal field theories in 2+1 dimensions: Fermionic Dirac matter coupled to U(1) gauge field, JHEP 01 (2015) 147 [Erratum ibid. 1603 (2016) 054] [arXiv: 1410.1902] [INSPIRE].

[21] S.M. Chester and S.S. Pufu, Anomalous dimensions of scalar operators in $Q E D_{3}$, JHEP 08 (2016) 069 [arXiv: 1603.05582] [INSPIRE].

[22] J. Polchinski, Renormalization and effective lagrangians, Nucl. Phys. B 231 (1984) 269 [INSPIRE].

[23] C. Wetterich, Exact evolution equation for the effective potential, Phys. Lett. B 301 (1993) 90 [INSPIRE].

[24] A.J. Niemi and G.W. Semenoff, Axial anomaly induced fermion fractionization and effective gauge theory actions in odd dimensional space-times, Phys. Rev. Lett. 51 (1983) 2077 [INSPIRE]. 
[25] A.N. Redlich, Gauge noninvariance and parity violation of three-dimensional fermions, Phys. Rev. Lett. 52 (1984) 18 [INSPIRE].

[26] A.N. Redlich, Parity violation and gauge noninvariance of the effective gauge field action in three-dimensions, Phys. Rev. D 29 (1984) 2366 [INSPIRE].

[27] M. Mulligan and F.J. Burnell, Topological insulators avoid the parity anomaly, Phys. Rev. B 88 (2013) 085104 [arXiv: 1301.4230] [INSPIRE].

[28] M. Franz, Z. Tesanovic and O. Vafek, $Q E D_{3}$ theory of pairing pseudogap in cuprates: From d-wave superconductor to antiferromagnet via 'algebraic' Fermi liquid, Phys. Rev. B 66 (2002) 054535 [cond-mat/0203333] [INSPIRE].

[29] D.J. Gross and A. Neveu, Dynamical symmetry breaking in asymptotically free field theories, Phys. Rev. D 10 (1974) 3235 [INSPIRE].

[30] J.A. Gracey, Electron mass anomalous dimension at $o\left(1 / n^{2}\right)$ in quantum electrodynamics, Phys. Lett. B 317 (1993) 415 [hep-th/9309092] [INSPIRE].

[31] R. Shankar, Renormalization group approach to interacting fermions, Rev. Mod. Phys. 66 (1994) 129 [INSPIRE]. 